\title{
AN IMPROVEMENT OF THE POINCARÉ-BIRKHOFF FIXED POINT THEOREM ${ }^{1}$
}

BY

\author{
PATRICIA H. CARTER
}

\begin{abstract}
If $g$ is a twist homeomorphism of an annulus $A$ in the plane which leaves at most one point in the interior of $A$ fixed, then there is an essential simple closed curve in the interior of $A$ which meets its image in at most one point; hence the annular region bounded by this simple closed curve and the inside component of the boundary of $A$ is mapped onto either a proper subset or a proper superset of itself.
\end{abstract}

1. Introduction. In 1912 Poincaré stated, and proved for some special cases, his Geometric Theorem for twist homeomorphisms of the annulus [16]:

If $g$ is an area preserving homeomorphism of the annulus $1 \leqslant r \leqslant 2$ onto itself which moves points on $r=1$ and $r=2$ in opposite angular directions to their new positions on $r=1$ and $r=2$ respectively, then $g$ has at least two distinct fixed points.

G. D. Birkhoff offered the first proof of this theorem in 1913 [3]; however, his proof of the existence of the second fixed point was incomplete. In 1925 Birkhoff announced [4] an extension of Poincaré's theorem in which he replaced the area preserving requirement by a more general topological condition, and no longer required the annulus to be the standard one, or the homeomorphism $g$ to be onto. This extension, which we will call the Poincaré-Birkhoff Fixed Point Theorem, may be stated as follows:

Suppose $A$ is an annulus in the plane bounded by $r=1$ and a simple closed curve $\gamma$ which lies in $r>1$ and intersects each radial in exactly one point. Suppose $g$ is a homeomorphism of $A$ onto the annulus $g(A)$, where $g(A)$ is bounded by $r=1$ and the simple closed curve $g(\gamma)$ which lies in $r>1$ and intersects each radial in exactly one point. If $g$ moves points on $r=1$ and $\gamma$ in opposite angular directions to their new positions on $r=1$ and $g(\gamma)$ respectively, then either there is a ring $S$ which has $r=1$ as its inner boundary and which is mapped onto a proper subset of itself by $g$ or $g^{-1}$, or else, $g$ has at least two fixed points.

\footnotetext{
Received by the editors August 21, 1979 and, in revised form, January 16, 1981. This result presented at the 1978 Annual Meeting of the AMS-MAA.

1980 Mathematics Subject Classification. Primary 55H25, 55M25, 55M20, 57N05.

Key words and phrases. Fixed point property, homeomorphism, twist homeomorphism of the annulus.

'The theorem proved in this paper is the main result of my dissertation of the same title. I would like to thank Dr. R. D. Mauldin for suggesting this problem, and for his advice and encouragement.

$I$ also thank the referee for carefully reading the manuscript.
} 
The most important difference between this theorem and Poincare's is the replacement of area preserving by the requirement that no ring with inner boundary $r=1$ is mapped onto a proper subset of itself by $g$ or $g^{-1}$. By a ring with inner boundary $r=1$ Birkhoff meant [4] a region bounded by $r=1$ and a continuum $C$ in $r \geqslant 1$, where the continuum $C$ has the property that it is the common boundary of a bounded, connected, simply connected open set and the closure of its complement. He did not require the continuum $C$ to be a simple closed curve. The requirement that $g$ move the points on the two components of the boundary of the annulus in opposite directions, that is, that $g$ be a twist homeomorphism, is ambiguous and needs a rigorous formulation (which is done for the standard annulus and the onto homeomorphism in [6]).

The topological condition in Birkhoff's theorem has frequently been misquoted in the literature; for example, in [18] the theorem is quoted as saying that either there is a simple closed curve that surrounds $r=1$ and does not intersect its image or there are two fixed points; and in [15] the theorem is quoted as saying if every simple closed curve surrounding $r=1$ intersects its image in at least two points, then there are two fixed points in the interior of $A$. In [7] the author gives an explicit example of a homeomorphism of a standard annulus for which the ring $S$, as constructed by Birkhoff in [4], has boundary the union of $r=1$ and a continuum $C$ which is not a simple closed curve. Further, the intersection of $C$ with its image contains a nondegenerate continuum.

Doubt about the correctness of Birkhoff's arguments for these theorems (especially about the correctness of his argument for the second fixed point) [2], [6], [17] has led to considerable effort towards finding convincing proofs. M. Brown and W. D. Neumann have given in [6] a precise and convincing proof, following Birkhoff's methods, for the area preserving theorem. In [10] and [11] Jacobowitz proves an area preserving version of the theorem more general than Poincarés, and applies it to finding solutions of certain differential equations. Several arguments have been given for the analogous theorem for area preserving flows on the standard annulus, see for example Abelson and Stanton [1], and Hermann [9]. The theorem has been generalized to a theorem about the number of components of the set of fixed points by W. D. Neumann in [14]. Proofs for the existence of at least one fixed point in the topological version of the theorem have been given by Kerékjarto [12] and by Barrar [2]; their lines of attack are quite different from Birkhoff's, and Barrar, in particular, finds a place for his version of the theorem in the context of a constellation of several important theorems of plane topology. Another method for proving the existence of at least one fixed point, using P.L. maps, can be found in Garcia [8].

An equivalent formulation of Birkhoff's topological version of the theorem is (briefly):

If $g$ is a twist homeomorphism of the annulus $A$ with at most one fixed point, then there is some ring $S$ with $r=1$ as its inner boundary which is mapped onto either $a$ proper subset or a proper superset of itself. 
The theorem I propose to prove is the following (a precise formulation of the hypothesis is given in the next section):

If $g$ is a twist homeomorphism of the annulus $A$ with at most one fixed point in the interior of $A$, then there is an essential simple closed curve $C$ in the interior of $A$ which intersects its image in at most one point.

The conclusion guarantees that the annulus bounded by $r=1$ and $C$ will be mapped onto either a proper subset or a proper superset of itself; thus this theorem implies Birkhoff's. There is no restriction on fixed points of $g$ on the boundary of $A$. One reason that this is desirable is because in Birkhoff's analysis of the billiard ball problem in [5] the (area preserving) twist homeomorphism of the annulus produced fixes every point of the boundary.

2. Statement of the theorem. Let $\gamma$ be a simple closed curve in the plane that lies in the exterior of the unit circle $T$ and intersects each radial in exactly one point. Let $A$ be the annulus bounded by $T$ and $\gamma$. Suppose $g$ is a homeomorphism of the annulus $A$ into the plane so that $g(T)=T$ and $g(\gamma)$ lies in the exterior of $T$ and intersects each radial in exactly one point. Let $\pi$ be the covering map from $\{(x, y)$ : $y>-1\}$ onto the punctured plane given by $\pi(x, y)=((1+y) \cos x,(1+y) \sin x)$, and let $\tilde{A}=\pi^{-1}(A)$. Since $\tilde{A}$ is the universal covering space of $A$ there is a homeomorphism $\tilde{g}$ from $\tilde{A}$ into the plane so that $g \circ \pi=\pi \circ \tilde{g}$, that is, so that $\tilde{g}$ makes the following diagram commute:

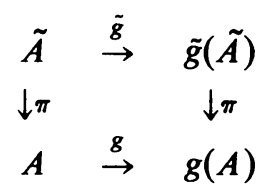

The homeomorphism $g$ is a twist homeomorphism if $\tilde{g}$ can be chosen so that $\tilde{g}$ moves points of one of $\pi^{-1}(T)$ and $\pi^{-1}(\gamma)$ to the left, and points of the other to the right. (We do not require $g$ to be fixed point free on $T \cup \gamma$.) A simple closed curve in $A$ is essential if it separates $T$ from $\gamma$.

THEOREM. If $g$ is a twist homeomorphism of the annulus $A$, and if $g$ has at most one fixed point in the interior of $A$, then there is an essential simple closed curve in the interior of $A$ which meets its image in at most one point. (If the curve found intersects its image, the point of intersection must be the fixed point of $g$ in the interior of $A$.)

This theorem cannot be improved in the direction of requiring the essential simple closed curve to miss its image. Figure 1 indicates a flow whose time $t$ map, for small positive $t$, is a twist homeomorphism which has one fixed point and no essential simple closed curve misses its image. ${ }^{2}$

${ }^{2}$ I thank the referee for suggesting this example. 


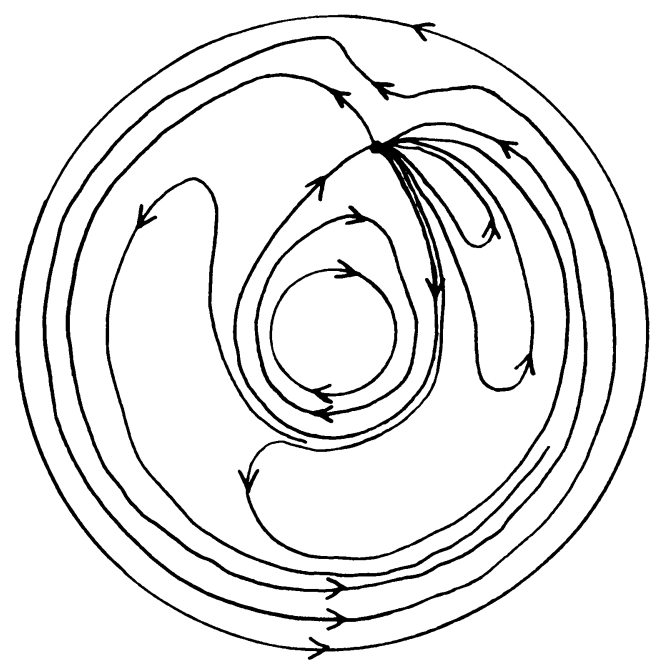

FIGURE 1

For $(a, b)$ and $(c, d)$ in the Cartesian plane $(a, b)+(c, d)=(a+c, b+d)$. The plane will be denoted by $E^{2}$, and the upper half-plane by $E^{+}$. The $\varepsilon$-neighborhood of the point $P$ will be denoted by $N(P, \varepsilon)$, and the $\varepsilon$-neighborhood of a set $B$, that is the set $\cup_{P \in B} N(P, \varepsilon)$, will be denoted by $N(B, \varepsilon)$. The usual distance between points $P$ and $Q$ will be denoted by $d(P, Q)$.

3. The homeomorphism $h$. If we let $F$ denote the continuous function from $R$ into $\mathcal{R}$ whose graph is $\pi^{-1}(\gamma)$, then $\tilde{A}=\{(x, y): 0 \leqslant y \leqslant F(x)\}$. Assume, without loss of generality, that $\tilde{g}$ moves points on $\pi^{-1}(T)$ to the right and points on $\pi^{-1}(\gamma)$ to the left; that is, for every $x, \tilde{g}(x, 0)=\left(x^{\prime}, 0\right)$ for some $x^{\prime}$ greater than $x$ and $\tilde{g}(x, F(x))=\left(x^{\prime \prime}, y\right)$ for some $x^{\prime \prime}$ less than $x$. Extend $\tilde{g}$ to a homeomorphism $h$ of the plane onto itself by requiring:

$$
h(x, y)= \begin{cases}\tilde{g}(x, y) & \text { if }(x, y) \text { is in } \tilde{A}, \\ \tilde{g}(x, 0)+(0, y) & \text { if } y<0, \\ \tilde{g}(x, F(x))+(0, y-F(x)) & \text { if } y>F(x) .\end{cases}
$$

From its definition we see that $h$ moves points of $y \leqslant 0$ to the right and points of $y \geqslant F(x)$ to the left; $h$ is periodic in the sense that $h(x+2 \pi, y)=h(x, y)+$ $(2 \pi, 0)$; and for $(x, y)$ in $\tilde{A}, h(x, y)=(x+2 n \pi, y)$, for some integer $n$ if and only if $\pi(x, y)$ is a fixed point of $g$. If $g$ is fixed point free in the interior of $A$, then $h$ is fixed point free in the whole plane. If $g$ has exactly one fixed point $W$ in the interior of $A$, then either $h$ has $\mathscr{F}=\pi^{-1}(W)$ as its set of fixed points or $h$ is fixed point free.

4. The minimal $\delta$-chain $P_{0}, P_{1}, \ldots, P_{N}$. Since $h$ is fixed point free on $y=0$ and on $y=F(x)$, and since $h$ is periodic, it follows that

$$
\xi=\frac{1}{3} \inf \left\{\left|x-x^{\prime}\right|:\left(x^{\prime}, 0\right)=h(x, 0) \text { or }\left(x^{\prime}, y\right)=h(x, F(x))\right\}
$$


is a positive number. There is a positive number $\mu$ less than $\xi$ so that for every point $P$ in the boundary of $\tilde{A}$, the diameter of $h(N(P, \mu))$ is less than $\xi$. Finally, there is a continuous, nonnegative function $\delta$ from $\tilde{A}$ into $\Re$ with the following properties:

(1) $\delta(x+2 \pi, y)=\delta(x, y)$,

(2) $\delta(P)=0$ if and only if $P$ is in $\mathscr{F}$,

(3) $\delta(P)<d(\mathscr{F}, P)$ if $P$ is not in $\mathscr{F}$,

(4) $\overline{N(P, 2 \delta(P))} \cap h \overline{N(P, 2 \delta(P))}=\varnothing$ if $P$ is not in $\mathscr{F}$,

(5) $\overline{N(P, 2 \delta(P))} \cap h^{-1} \overline{N(P, 2 \delta(P))}=\varnothing$ if $P$ is not in $\mathscr{F}$, and

(6) $\delta(P)<\mu$.

Extend $\delta$ to all of $y \geqslant 0$ by requiring $\delta(x, y)=\delta(x, F(x))$ for $y \geqslant F(x)$. Observe that if $h$ is fixed point free then there is a constant map $\delta$ with these properties.

A $\delta$-chain $P_{0}, P_{1}, \ldots, P_{N}$ is a finite sequence of points from $y \geqslant 0$ with $P_{0}$ in $y=0, P_{k}$ in $\tilde{A}$ for $0<k<N$, and $d\left(P_{k+1}, h\left(P_{k}\right)\right)<\delta\left(h\left(P_{k}\right)\right)$ for $0 \leqslant k<N$. A $\delta$-chain $P_{0}, P_{1}, \ldots, P_{N}$ is terminating if $P_{N}$ is in $y \geqslant F(x)$.

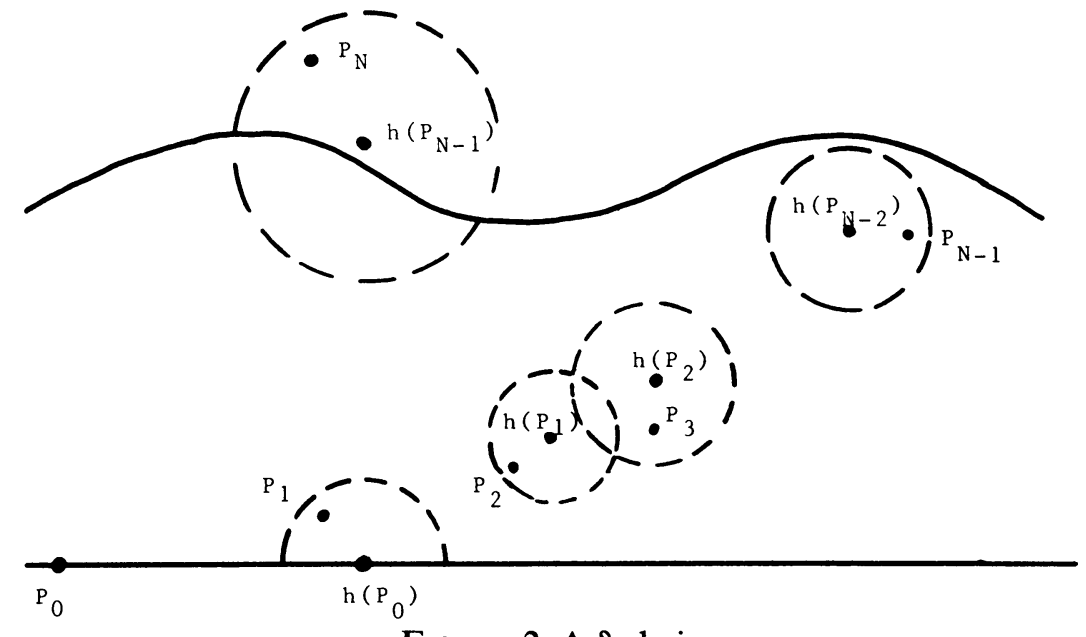

FIGURE 2. A $\delta$-chain

For each nonnegative integer $n$, let $V_{n}$ be the set of all points $P$ such that there is a $\delta$-chain $P_{0}, P_{1}, \ldots, P_{n}$ with $P=P_{n}$. It follows immediately that $V_{0}$ is $y=0$, and for positive $n, V_{n}=\bigcup\left\{N(P, \delta(P)) \cap E^{+}: P \in h\left(\tilde{A} \cap V_{n-1}\right)\right\}$. If $P_{0}, P_{1}, \ldots, P_{n}$ is a $\delta$-chain, then $h^{-1}\left(P_{0}\right), P_{0}, P_{1}, \ldots, P_{n}$ is a $\delta$-chain; hence $V_{n} \subset V_{n+1}$. The sets $V_{n}$ are periodic in the sense that $V_{n}+(2 \pi, 0)=V_{n}$. Let $V=\cup_{n=0}^{\infty} V_{n}$. If we let $\varepsilon$ be the minimum of $\delta$ on $y=0$, and $\eta$ be the maximum of $\delta$ on $E^{+}$, then both $\varepsilon$ and $\eta$ are positive, and $\{(x, y): 0 \leqslant y<\varepsilon\} \subset V_{1} \subset V \subset N(\tilde{A}, \eta)$. Now let $M_{n}=$ $\pi\left(V_{n}\right)$ and $M=\cup_{n=0}^{\infty} M_{n}$; then $M_{0}=T, M_{n} \subset M_{n+1}, \pi(V)=M$ and $\{(r, \theta)$ : $1 \leqslant r<1+\varepsilon\} \subset M_{1} \subset M \subset N(A, \eta)$.

Let $B$ be the unbounded component of the complement of $\bar{M}$; the open unit disk $U$ is one of the bounded components of the complement of $\bar{M}$. If we let $S$ be the complement of $\bar{B} \cup U$, then $M \subset S \subset N(A, \eta)$ and the set $S \cup U$ is open and bounded.

LEMMA 4.1. If there is no terminating $\delta$-chain, then $S$ is homeomorphic to the half-open annular region $\{(r, \theta): 1 \leqslant r<2\}$, and $g(S) \subset S \subset A$. 
Proof. Suppose there is no terminating $\delta$-chain. Since under this assumption $h\left(V_{n-1}\right)$ is a subset of $\tilde{A}$, it follows that, for positive $n$,

$$
V_{n}=\bigcup\left\{N(P, \delta(P)) \cap E^{+}: P \in h\left(V_{n-1}\right)\right\}
$$

This characterization shows that $V_{n}$ is connected, and $V_{n} \backslash\{(x, y): y=0\}$ is open and connected. Since $V$ is the increasing union of connected sets it is connected, and since $V \backslash\{(x, y): y=0\}$ is the increasing union of open connected sets it is open and connected. Thus, since $M$ is connected and $M \backslash T$ is open and connected, $M \cup U$ is open and connected. Since $S \cup U$ is the interior of the closure of the set that consists of the union of $\overline{M \cup U}$ and the bounded components of its complement, $S \cup U$ is connected. It is simply connected because its complement $\bar{B}$ is connected. Since $S \cup U$ is open, bounded, connected and simply connected it is homeomorphic to the open unit disk. This, together with the fact that $\{(r, \theta)$ : $1 \leqslant r<1+\varepsilon\} \subset S$, implies $S$ is homeomorphic to the half-open annular region $\{(r, \theta): 1 \leqslant r<2\}$.

Since $V_{n} \subset \tilde{A}$ it follows that $M_{n} \subset A$ for every $n$; thus $M \subset A$. Hence $B$ includes the unbounded component of $E^{2} \backslash \gamma$, and therefore $S$ is a subset of $A$. Since $h\left(V_{n}\right) \subset V_{n+1}$, and therefore, $g\left(M_{n}\right)=g \circ \pi\left(V_{n}\right)=\pi \circ h\left(V_{n}\right) \subset \pi\left(V_{n+1}\right)=$ $M_{n+1}$ it follows that $g(M) \subset M$. Thus, since $E^{2} \backslash \bar{M} \subset g\left(E^{2} \backslash \bar{M}\right)$ and $B$ is the only unbounded component of $E^{2} \backslash \bar{M}$, it follows that $\bar{B} \subset g(\bar{B})$; therefore $g(S) \subset$ $S$.

The boundary of $S$ has exactly two components; let $\beta S$ denote that component which is not $T$. The continuum $\beta S$ is a subset of the interior of $A$ which separates $T$ from $\gamma$ but it may not be a simple closed curve.

LEMMA 4.2. If there is no terminating $\delta$-chain, then there is an essential simple closed curve in the interior of $A$ which meets its image in at most one point.

Proof. Let $W$ be the only fixed point of $g$ in the interior of $A$ (if any). First we show that $g$ maps $\beta S$ into $S$ except perhaps for the point $W$. Let $X$ be any point in $\beta S$ different from $W$, and let $P$ be in $\pi^{-1}(X)$; in this case $\delta(h(P))>0$. Since $P$ is a limit point of $V$ there is a point $Q$ in $V$ close enough to $P$ so that both $\delta(h(Q)) \geqslant \frac{3}{4} \delta(h(P))$ and $d(h(P), h(Q))<\frac{1}{4} \delta(h(P))$. If $n$ is a positive integer so that $Q$ is in $V_{n}$, then $h(Q)$ is in $V_{n+1}$. From the assumption that no $\delta$-chain terminates it follows that

$$
N(h(Q), \delta(h(Q))) \cap E^{+} \subset V_{n+1} \subset \pi^{-1}(S) .
$$

Thus since $h(P)$ is in $N(h(Q), \delta(h(Q)))$, it follows that $h(P)$ is in $\pi^{-1}(S)$. Hence $g(X)$ is in $S$.

If $g$ has no fixed points in the interior of $A$, then $\beta S$ and $g(\beta S)$ are disjoint continua, and thus can be separated by a simple closed curve $\psi$. The curve $\psi$ is an essential simple closed curve in the interior of $A$ which does not intersect its image.

If $g$ has the one fixed point $W$ in the interior of $A$ then either $\beta S$ and $g(\beta S)$ are disjoint, in which case the argument of the last paragraph applies, or else $\beta S \cap$ $g(\beta S)=\{W\}$. In the latter case, since each of $\beta S$ and $g(\beta S)$ is the common boundary of a bounded, connected, simply connected open set and its complement, 
each of $\beta S \backslash\{W\}$ and $g(\beta S) \backslash\{W\}$ is connected. Therefore the continua $\beta S$ and $g(\beta S)$ satisfy the hypotheses of Theorem 44 of [13] which states:

If $H$ and $K$ are continua in the plane such that $K$ does not separate any two points of $H, H \cap K$ is totally disconnected, and $K \backslash H$ is connected, then there is a simple closed curve which separates $K \backslash H$ from $H \backslash K$ and contains $H \cap K$ but no other points of $H$ or $K$.

Thus, there is a simple closed curve $\psi$ which separates $\beta S \backslash\{W\}$ from $g(\beta S) \backslash\{W\}$ and contains $W$. The curve $\psi$ is an essential simple closed curve in the interior of $A$ which intersects its image in exactly one point.

In the sequel we assume that there is some terminating $\delta$-chain for $g$.

Choose a terminating $\delta$-chain $P_{0}, P_{1}, \ldots, P_{N}$ of minimal length $N$. As a result of this minimality requirement this particular terminating $\delta$-chain has the following additional properties: The points $P_{0}, P_{1}, \ldots, P_{N-1}$, and $P_{N}$ are distinct, and so are their images under $h$; $h\left(P_{i}\right)$ may be $P_{j}$ only if $j=i+1$. If $i>j+1$, then $P_{i}$ is not in $N\left(h\left(P_{j}\right), \delta\left(h\left(P_{j}\right)\right)\right)$; if $i<N-1$, then $N\left(h\left(P_{i}\right), \delta\left(h\left(P_{i}\right)\right)\right)$ is a subset of $y<F(x)$.

5. The isotopy $\Phi$. In this section we give an isotopy $\Phi$ of the plane for which $\Phi(\cdot, 0)$ is the identity, $\Phi(\cdot, 1)$ moves $h\left(P_{i-1}\right)$ to $P_{i}$, and $\Phi(\cdot, t)$ is the identity on the complement of the union of a neighborhood of $y=0$ and a set of small disjoint topological disks $D_{i}$ containing the points $h\left(P_{i-1}\right)$ and $P_{i}$.

For $1 \leqslant i \leqslant N$, let $G_{i}=N\left(h\left(P_{i-1}\right), \delta\left(h\left(P_{i-1}\right)\right)\right) \cap\{(x, y): y>0\}$. Note that for $1 \leqslant i<N, G_{i}$ is contained in $\tilde{A}$.

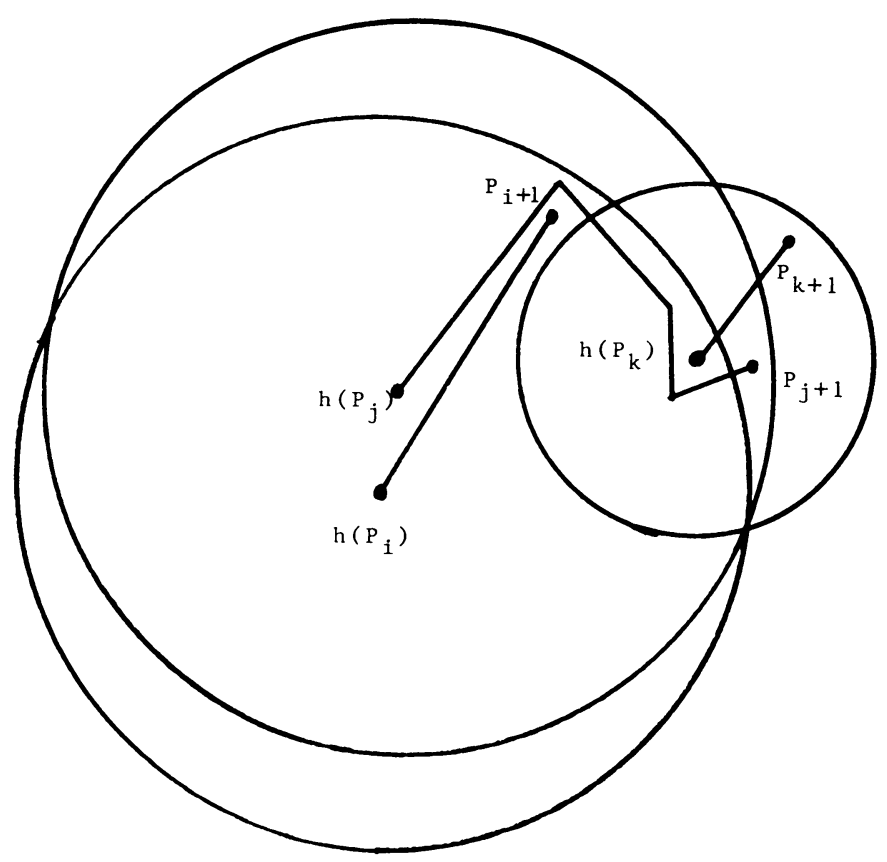

FigURE 3 
LEMMA 5.1. There is a collection $\left\{\alpha_{i}: 1 \leqslant i \leqslant N\right\}$ of pairwise disjoint arcs so that each $\alpha_{i}$ is a simple (or degenerate) polygonal arc in $G_{i}$ from $h\left(P_{i-1}\right)$ to $P_{i}$.

Proof. If $k$ is less than $N$, a set $\left\{\eta_{i}: 1 \leqslant i \leqslant k\right\}$ satisfying the lemma will be called a set of connecting arcs for the subchain $P_{0}, P_{1}, \ldots, P_{k}$ of $P_{0}, P_{1}, \ldots, P_{N}$. By minimality $P_{1}$ must be in $y>0$. Let $\eta_{1}$ be a simple polygonal arc from $h\left(P_{0}\right)$ to $P_{1}$ in $G_{1} \cup\left\{h\left(P_{0}\right)\right\}$ which misses every point of $\left\{Q: Q=h\left(P_{i}\right)\right.$ or $P_{i+1}, 1 \leqslant i<$ $N\}$. The arc $\eta_{1}$ is a connecting arc for the subchain $P_{0}, P_{1}$.

Suppose now that for $k$ less than $N$ a set of connecting arcs $\left\{\eta_{i}: 1 \leqslant i \leqslant k\right\}$ has been constructed for $P_{0}, P_{1}, \ldots, P_{k}$, with the additional property that:

$$
\bigcup_{i=1}^{k} \eta_{i} \cap\left\{Q: Q=h\left(P_{i}\right) \text { or } P_{i+1} \text { for } k \leqslant i<N\right\}=\varnothing
$$

It remains to show there is a set of connecting arcs for $P_{0}, P_{1}, \ldots, P_{k}, P_{k+1}$.

If $P_{k+1}=h\left(P_{k}\right)$, then let $\eta_{k+1}$ be the map whose image is $h\left(P_{k}\right)$. The set $\left\{\eta_{i}\right.$ : $1 \leqslant i \leqslant k+1\}$ is a set of connecting arcs for $P_{0}, P_{1}, \ldots, P_{k}, P_{k+1}$.

Now suppose $P_{k+1} \neq h\left(P_{k}\right)$. The set $\cup\left\{\eta_{i}: 1 \leqslant i \leqslant k\right\} \cap G_{k+1}$ has at most finitely many components, each with closure a polygonal arc. Let $B_{1}$ be the set of all the components of $\cup\left\{\eta_{i}: 1 \leqslant i \leqslant k\right\} \cap G_{k+1}$ that do not separate $P_{k+1}$ from $h\left(P_{k}\right)$ in $G_{k+1}$, and let $B_{2}$ be the set of all the components that do. Let $V$ be the component of

$$
G_{k+1} \backslash\left(\cup B_{1} \cup\left\{Q: Q=h\left(P_{i}\right) \text { or } P_{i+1} \text { for } k+1 \leqslant i<N\right\}\right)
$$

that contains $h\left(P_{k}\right)$ and $P_{k+1}$. The elements of $B_{2}$ may be labeled $\beta_{1}, \beta_{2}, \ldots, \beta_{l}$ in such a way that $\beta_{i}$ separates $h\left(P_{k}\right)$ from $\beta_{j}$ in $V$ if and only if $i<j$. Let the map $f$ from $\{1,2, \ldots, l\}$ into $\{1,2, \ldots, k\}$ be defined by: $f(i)=j$ if and only if $\beta_{i}$ is a subset of $\eta_{j}$. The set $V$ is a topological disk minus at most finitely many points and contains $\cup B_{2}$. Since $\beta_{i}$ is a subset of $G_{f(i)}$ which separates $P_{k+1}$ from $h\left(P_{k}\right)$ in $V$, and since $P_{k+1}$ is not in $G_{f(i)}$ by the minimality of the length of $P_{0}, P_{1}, \ldots, P_{N}$, from the geometry it follows that $h\left(P_{k}\right)$ is in $G_{f(i)}$ and a component of $\partial G_{f(i)} \cap V$ separates $h\left(P_{k}\right)$ from $P_{k+1}$.

There is a simple polygonal arc $\sigma$ in $V$ from $h\left(P_{k}\right)$ to $P_{k+1}$ so that, for each $i, \sigma$ intersects each of $\beta_{i}$ and $\partial G_{f(i)}$ in exactly one point and the subarc of $\sigma$ from $h\left(P_{k}\right)$ to $\sigma \cap \beta_{i}$ is contained in $G_{f(i)}$. There is a polygonal simple closed curve $\zeta$ in $V$ so that, for each $i, \zeta$ intersects each of $\beta_{i}$ and $\partial G_{f(i)}$ in exactly two points, and so that the topological disk $D$ bounded by $\zeta$ contains $\sigma$ in its interior. The arc $\beta_{i} \cap D$ separates $h\left(P_{k}\right)$ from the arc $\partial G_{f(i)} \cap D$, and the component of $D \backslash \partial G_{f(i)}$ containing $h\left(P_{k}\right)$ is a subset of $G_{f(i)}$. There is a homeomorphism $\Upsilon$ of $D$ onto itself that is the identity on $\zeta$, and maps each $\beta_{i} \cap D$ onto a simple polygonal arc in $(D \backslash \sigma) \cap G_{f(i)}$. For each $i$, let $\beta_{i}^{\prime}$ be the simple polygonal arc obtained from $\beta_{i}$ by replacing $\beta_{i} \cap D$ with its image under $\Upsilon$. If we let $\eta_{j}^{\prime}$ be the polygonal arc resulting from the replacement of $\beta_{i}$ by $\beta_{i}^{\prime}$ for each $\beta_{i}$ in $B_{2}$ that is a subset of $\eta_{j}$ (if any), then $\left\{\eta_{i}^{\prime}\right.$ : $1 \leqslant i \leqslant k\} \cup\{\sigma\}$ is a set of connecting arcs for $P_{0}, P_{1}, \ldots, P_{k}, P_{k+1}$. Hence, by induction, there is a set of connecting arcs $\left\{\alpha_{i}: 1 \leqslant i \leqslant N\right\}$ for $P_{0}, P_{1}, \ldots, P_{N}$. 

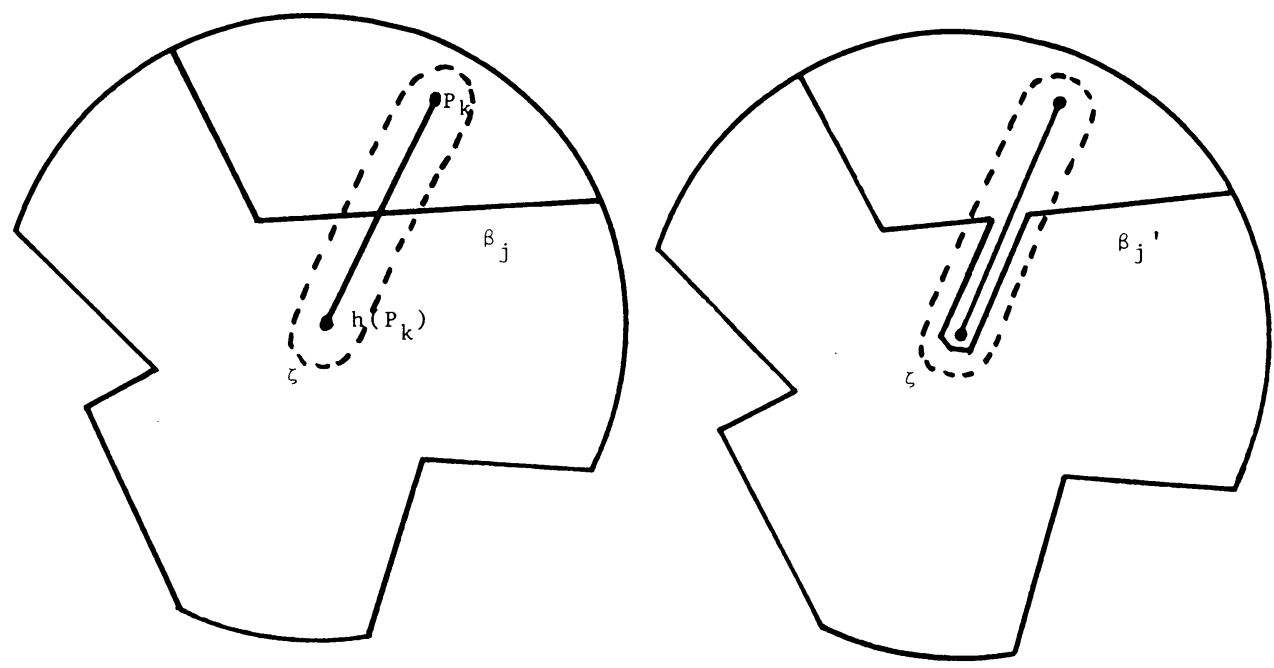

FIGURE 4

For $1<i \leqslant N$ there are disjoint topological disks $D_{i}$ so that $\alpha_{i}$ is in the interior of $D_{i}, D_{i}$ is disjoint from $\alpha_{1}, D_{N}$ is a subset of $G_{N}$, and, for $1<i<N, D_{i}$ is a subset of $G_{i}$ and hence also of $\{(x, y): 0<y<F(x)\}$. There is a positive number $\nu$, numbers $x_{1}$ and $x_{2}$, and a topological disk $D_{1}$, so that $\nu$ is less than each of the minimum value of $\delta$ on $y=0$ and half the distance from $\cup_{i=2}^{N} D_{i}$ to $y=0$, so that the disk $D_{1}$ is a subset of $N\left(h\left(P_{0}\right), \delta \circ h\left(P_{0}\right)\right)$ that contains $\alpha_{1}$ in its interior and is disjoint from $\cup_{i=2}^{N} D_{i}$ and, further, so that $\partial D_{1} \cap\{(x, y): y<2 \nu\}$ is the union of $\left\{(x, y):-2 \nu \leqslant y \leqslant 2 \nu\right.$ and $x=x_{1}$ or $\left.x=x_{2}\right\}$ and $\left\{(x, y): x_{1} \leqslant x \leqslant x_{2}\right.$ and $y=-2 \nu\}$. Let $D_{0}$ be the divided strip $\left\{(x, y): x \leqslant x_{1}\right.$ or $x \geqslant x_{2}$, and $-2 \nu \leqslant y \leqslant$ $2 v\}$.

If $a$ and $b$ are two points in the interior of a topological disk $D$, then there is a continuous map $\Phi$ from $D \times[0,1]$ onto $D$ so that $\Phi(\cdot, t)$ is a homeomorphism of $D$ onto $D$ for each $t$ in $[0,1], \Phi(P, 0)=P$ for all $P$ in $D, \Phi(P, t)=P$ for all $P$ in $\partial D$ and all $t$ in $[0,1]$, and $\Phi(a, 1)=b$. Applying this to each of the sets $D_{i}$, for $1<i \leqslant N$, there is a continuous map $\Phi_{i}$ from $D_{i} \times[0,1]$ onto $D_{i}$ so that $\Phi_{i}(\cdot, t)$ is a homeomorphism of $D_{i}$ onto itself for each $t$ in $[0,1], \Phi_{i}(P, 0)=P$ for every $P$ in $D_{i}, \Phi_{i}(P, t)=P$ for every $P$ in $\partial D_{i}$ and all $t$ in $[0,1]$, and $\Phi_{i}\left(h\left(P_{i-1}\right), 1\right)=P_{i}$.

Let $f$ be the homeomorphism of $[-2 \nu, 2 \nu]$ onto itself which maps $[-2 \nu,-\nu]$ linearly onto $[-2 \nu, 0],[-\nu, 0]$ linearly onto $[0, \nu]$, and $[0,2 \nu]$ linearly onto $[\nu, 2 \nu]$. Let $\Phi_{0}$ be the continuous map from $D_{0} \times[0,1]$ onto $D_{0}$ given by $\Phi_{0}((x, y), t)=$ $(x,(1-t) y+t f(y))$. For each $t$ in $[0,1], \Phi_{0}(\cdot, t)$ is a homeomorphism of $D_{0}$ onto itself, $\Phi_{0}(P, 0)=P$ for every $P$ in $D_{0}$, and $\Phi_{0}(P, t)=P$ for all $P$ in $y=-2 \nu$ or $y=2 \nu$ and all $t$ in $[0,1]$. There is a continuous map $\Phi_{1}$ from $D_{1} \times[0,1]$ into $D_{1}$ so that, for all $t$ in $[0,1], \Phi_{1}(\cdot, t)$ is a homeomorphism of $D_{1}$ onto itself, $\Phi_{1}(P, 0)=P$ for all $P$ in $D_{1}, \Phi_{1}(P, t)=P$ for all $P$ in $\partial D_{1} \cap\{(x, y): y \geqslant 2 \nu\}$ and all $t$ in [0,1], $\Phi_{1}((x, y), t)=(x,(1-t) y+t f(y))$ for $(x, y)$ in $D_{0} \cap D_{1}$ or $\left\{(x, y): x_{1} \leqslant x<x_{2}\right.$, $-2 \nu \leqslant y \leqslant-\nu\}$, and $\Phi_{1}\left(h\left(P_{0}\right), 1\right)=P_{1}$. 
Let $\Phi$ be the continuous map from $E^{2} \times[0,1]$ onto $E^{2}$ defined by $\Phi(P, t)=$ $\Phi_{i}(P, t)$ if $P$ is in $\cup_{i=0}^{N} D_{i}$ and $\Phi(P, t)=P$ if $P$ is not in $\cup_{i=0}^{N} D_{i}$. For every $t$ in $[0,1], \Phi(\cdot, t)$ is a homeomorphism of the plane onto itself which is the identity on the complement of $\cup_{i=0}^{N} D_{i}$. The homeomorphism $\Phi(\cdot, 0)$ is the identity, and $\Phi(\cdot, 1)$ maps $h\left(P_{i-1}\right)$ to $P_{i}$ and the infinite strip $-\nu \leqslant y \leqslant 0$ onto an infinite strip bounded below by $y=0$.

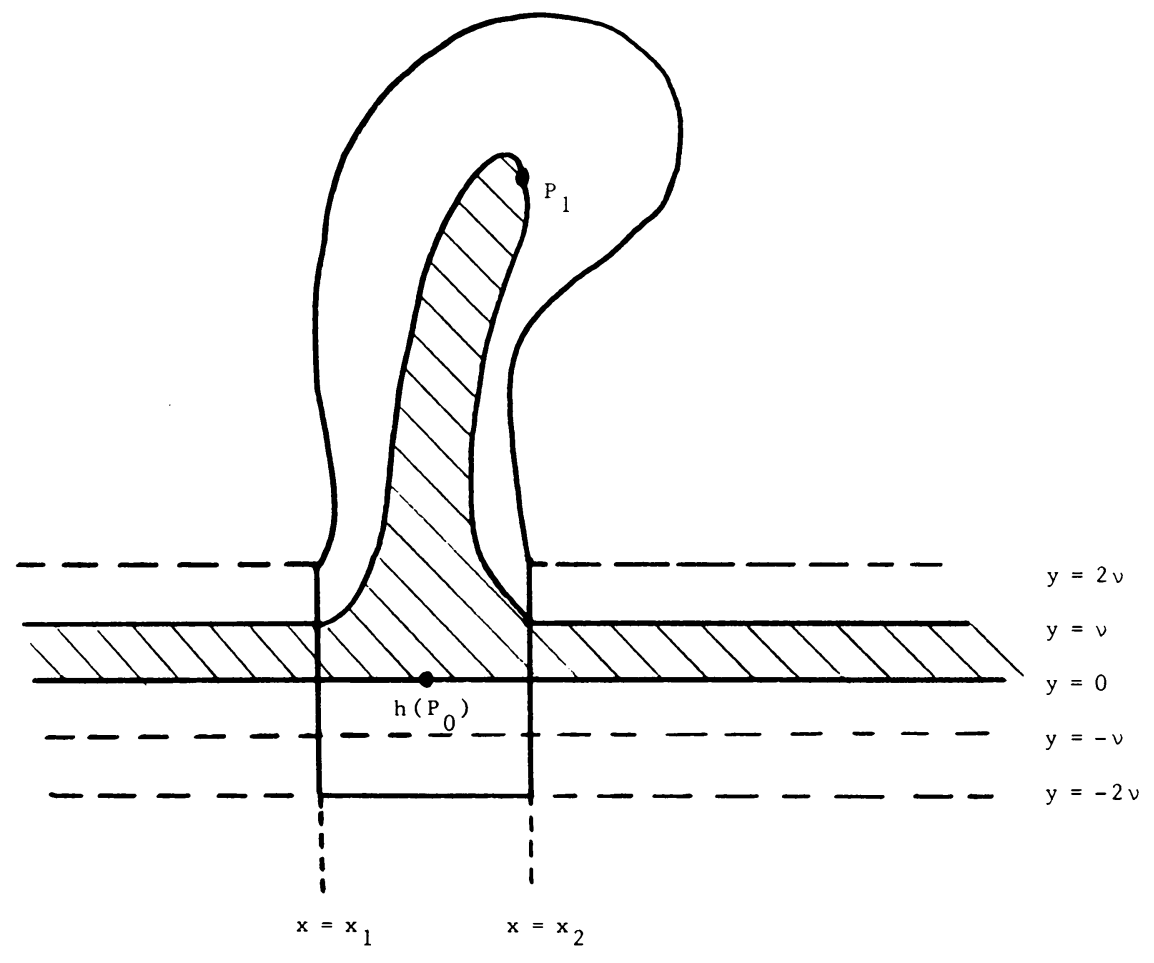

Figure 5. The image of $-\nu \leqslant y \leqslant 0$ under $\Phi(\cdot, 1)$

6. The homeomorphism $h_{1}$. Let $H$ be the continuous map from $E^{2} \times[0,1]$ onto $E^{2}$ defined by $H(P, t)=\Phi(h(P), t)$. For every $t$ in $[0,1], H(\cdot, t)$ is a homeomorphism of the plane onto itself that moves points in $y<0$ to the right and points in $y \geqslant F(x)$ to the left. Since $\Phi(\cdot, 0)$ is the identity $H(\cdot, 0)=h$. For each $t$ in $[0,1]$ let $h_{t}=H(\cdot, t)$.

LEMMA 6.1. For every $t$ in $[0,1]$ the homeomorphism $h_{t}$ has the same fixed points as $h$.

Proof. Let $D=\cup_{i=0}^{N} D_{i}$. If $Q$ is in $E^{2} \backslash h_{t}^{-1}(D)$, then $h_{t}(Q)$ is in $E^{2} \backslash D$. Since $\Phi$ is the identity on $E^{2} \backslash D, h_{t}(Q)=\Phi(h(Q), t)=h(Q)$; thus $h_{t}$ has exactly the same fixed points as $h$ in $E^{2} \backslash h_{t}^{-1}(D)$.

If $Q$ is in $h_{t}^{-1}(D)$, then $h_{t}(Q)$ is in $D$. The homeomorphism $h^{-1}$ has no fixed points in $D$; to show that $h_{t}(Q) \neq Q$ for $Q$ in $h_{t}^{-1}(D)$ there are two cases to 
consider. First, if $h_{t}(Q)$ is in $D_{i}$ for some positive $i$, then $h_{t}(Q)$ is in $N\left(h\left(P_{i-1}\right), \delta \circ h\left(P_{i-1}\right)\right) ; h(Q)$ is in this neighborhood of $h\left(P_{i-1}\right)$ by the construction of $\Phi$, but $Q$ is not since $\delta$ was chosen so that

$$
\overline{N(P, 2 \delta(P))} \cap h^{-1} \overline{N(P, 2 \delta(P))}=\varnothing
$$

for $P$ not a fixed point of $h$; therefore, $h_{t}(Q) \neq Q$. If $h_{t}(Q)$ is in $D_{0}$, then by the choice of $\nu$ and $\delta, d\left(h_{t}(Q), h(Q)\right)<\frac{1}{2} d(h(Q), Q)$; therefore, $h_{t}(Q) \neq Q$ so that $h_{t}$ has the same fixed points as $h$ in $h_{t}^{-1}(D)$. Thus $h_{t}$ has exactly the same fixed points as $h$.

7. The simple arc $C$. Let $P_{-1}=h_{1}^{-1}\left(P_{0}\right)$. If $k$ is an integer with $-1 \leqslant k<N$, then $h_{1}\left(P_{k}\right)=P_{k+1}$. Define the continuous map $C$ from $[-1, N+1]$ into the plane inductively: let $C:[-1,0] \rightarrow E^{2}$ be the straight line segment from $P_{-1}$ to $P_{0}$, and for each nonnegative integer $k$ let $C:[k, k+1] \rightarrow E^{2}$ by $C(t)=h_{1}(C(t-1))$.

Let $U_{0}=\{(x, y):-\nu<y \leqslant 0\}$, and for each $i$ with $i \geqslant-1$ let $U_{i}=h_{1}^{i}\left(U_{0}\right)$. Since $U_{-1} \cap U_{0}=\varnothing$ and $\bar{U}_{-1}$ is separated from $\bar{U}_{1}$ by $y=-\nu / 2$, therefore by induction: if $i \neq j$, then $U_{i} \cap U_{j}=\varnothing$, and if $|i-j|>1$, then $\bar{U}_{i} \cap \bar{U}_{j}=\varnothing$.

LEMMA 7.1. The continuous map $C$ from $[-1, N+1]$ into the plane is a simple arc with $C(k)=P_{k}$.

Proof. Since $C((0,1])$ is a subset of $U_{0}$, for each positive integer $k, C((k-1, k])$ $=h_{1}^{k}(C((-1,0]))$ is a subset of $U_{k}$. Thus, if $k \neq k^{\prime}$, then

$$
C((k-1, k]) \cap C\left(\left(k^{\prime}-1, k^{\prime}\right]\right)=\varnothing ;
$$

and if $\left|k-k^{\prime}\right|>1$, then $C([k-1, k]) \cap C\left(\left[k^{\prime}-1, k^{\prime}\right]\right)=\varnothing$. Thus $C$ is a simple arc.

Since $C(0)=P_{0}$, and $C(k)=h_{1}(C(k-1))$, by induction, $C(k)=P_{k}$ for all integers $k$ with $-1 \leqslant k \leqslant N$.

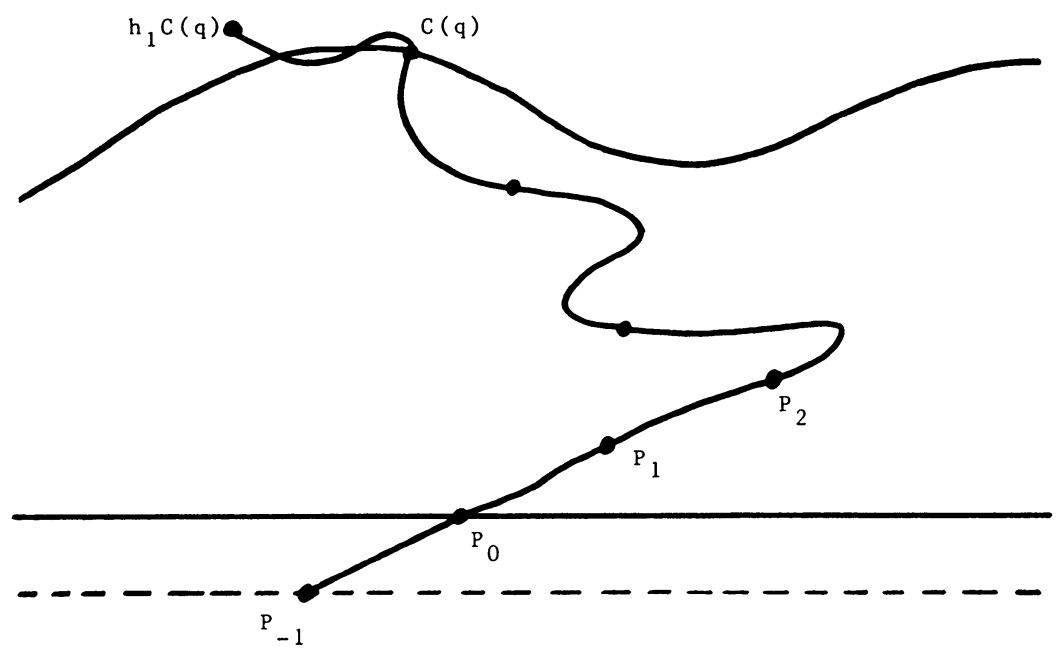

Figure 6. The simple curve $C^{*}$ 
LEMMA 7.2. There is a number $q$ in $(N-1, N]$ so that $C([-1, q])$ crosses $\tilde{A}$ and intersects $y=F(x)$ in exactly one point $Q=C(q)$.

Proof. Since $C([-1, N])$ contains the point $P_{0}$ of $y \leqslant 0$ and the point $P_{N}$ of $y \geqslant F(x), C([-1, N])$ must intersect $y=F(x)$. Let $q$ be the first number in $[-1, N]$ for which $Q=C(q)$ is in $y=F(x)$. Let the sets $V_{i}$ be defined as in $\S 4$. Since $U_{1} \subset V_{1}$ and $\Phi\left(h\left(V_{i}\right), 1\right) \subset V_{i+1}$ for $1 \leqslant i<N$, by induction:

$$
\begin{aligned}
C((k-1, k]) & \subset U_{k}=h_{1}\left(U_{k-1}\right)=\Phi\left(h\left(U_{k-1}\right), 1\right) \\
& \subset \Phi\left(h\left(V_{k-1}\right), 1\right) \subset V_{k} \subset V_{N-1}
\end{aligned}
$$

for $0<k \leqslant N-1$. Thus $C((0, N-1]) \subset V_{N-1}$. Since the $\delta$-chain $P_{0}, P_{1}, \ldots, P_{N}$ is of minimal length, $V_{N-1}$ does not intersect $y=F(x)$; thus $C([-1, N-1])$ does not intersect $y=F(x)$. Hence $N-1<q \leqslant N$.

Let $C^{*}$ be the map $C$ restricted to $[-1, q]$.

8. Using the index of curves crossing $\tilde{A}$ to obtain a contradiction. Suppose $f$ is a homeomorphism of the plane onto itself and $\alpha$ is a continuous map of the interval $[a, b]$ into the plane so that $f$ has no fixed points on $\alpha([a, b])$. As $t$ varies from $a$ to $b$ the vector from $P=\alpha(t)$ to $f(P)=f(\alpha(t))$ rotates; the index is a precise way of measuring this rotation. Let $D(P, Q)$ be the unit vector in the same direction as $Q-P$, that is, $D(P, Q)=(Q-P) /\|Q-P\|$. Let $\bar{f}$ be the map from $\alpha([a, b])$ into $S^{1}$ given by $\bar{f}(P)=D(P, f(P))$. If $\pi$ is the covering map from $\Re$ to $S^{1}$ given by $\pi(x)=(\cos x, \sin x)$, then there is a map $\tilde{\alpha}$ from $[a, b]$ into $R$ so that $\pi \circ \tilde{\alpha}=\bar{f} \circ \alpha$, that is so that the following diagram commutes:

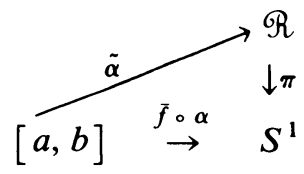

Define the index of the curve $\alpha$ under the homeomorphism $f$ by

$$
\operatorname{Ind}[f, \alpha]=(\tilde{\alpha}(b)-\tilde{\alpha}(a)) / 2 \pi \text {. }
$$

For details about the properties of the index see [6] or [14]. The remainder of the argument is similar to corresponding arguments in [4], [6], and [14]; so some details have been omitted.

LEMMA 8.1. If $P$ is a point of $y \leqslant 0$, and $Q$ is a point of $y \geqslant F(x)$, then for any arc $\alpha$ from $P$ to $Q$ containing no fixed points of $h, 1 / 8<\operatorname{Ind}\left[h_{t}, \alpha\right]<3 / 4 \bmod 1$.

Proof. Let $\theta_{1}$ be the angle from $\overrightarrow{P, P+(1,0)}$ to $\overrightarrow{P, h_{t}(P)}$, and let $\theta_{2}$ be the angle from $\overrightarrow{Q, Q+(1,0)}$ to $\overrightarrow{Q, h_{t}(Q)}$ (both counterclockwise). Since the angles $\theta_{1}$ and $\theta_{2}$ satisfy $0 \leqslant \theta_{1}<\pi / 4$, and $\pi / 2<\theta_{2}<3 \pi / 2$, we have $\pi / 4<\theta_{2}-\theta_{1}<3 \pi / 2$. Since $\operatorname{Ind}\left[h_{t}, \alpha\right]=\left(\theta_{2}-\theta_{1}\right) / 2 \pi \bmod 1$, this implies that $1 / 8<\operatorname{Ind}\left[h_{t}, \alpha\right]<3 / 4$ $\bmod 1$.

LEMMA 8.2. If $P$ is a point in $y \leqslant 0$ and $Q$ is a point in $y>F(x)$, then $\operatorname{Ind}[h, \alpha]$ is the same for every arc $\alpha$ from $P$ to $Q$ that contains no fixed points of $h$. 
Proof. The proof of this is essentially the same as given on p. 24 of [6] and so is omitted.

LEMMA 8.3. For the arc $C^{*}, 1 / 8<\operatorname{Ind}\left[h_{1}, C^{*}\right]<3 / 4$.

Proof. Let $\theta_{1}$ be the counterclockwise angle from $\overrightarrow{P_{-1}, P_{-1}+(1,0)}$ to $\overrightarrow{P_{-1}, h_{1}\left(P_{-1}\right)}$, and let $\theta_{2}$ be the counterclockwise angle from $\overrightarrow{Q, Q+(1,0)}$ to $Q, h_{1}(Q)$. Since $C^{*}$ is a flow line for $h_{1}$ a homotopy essentially the same as the one given on pp. 29-30 of [6] can be defined to show that $\operatorname{Ind}\left[h_{1}, C^{*}\right]=\left(\theta_{2}-\theta_{1}\right) / 2 \pi$. The lemma follows from the fact that $1 / 8<\left(\theta_{2}-\theta_{1}\right) / 2 \pi<3 / 4$.

LEMMA 8.4. The following inequality holds for the curve:

$$
1 / 4<\operatorname{Ind}\left[h, C^{*}\right]<3 / 4 \text {. }
$$

Proof. For each $t$ in $[0,1]$, let $\theta_{1}^{t}$ be the counterclockwise angle from $\overrightarrow{P_{-1}, P_{-1}+(1,0)}$ to $\overrightarrow{P_{-1}, h_{t}\left(P_{-1}\right)}$ and let $\theta_{2}^{t}$ be the counterclockwise angle from $\overline{Q, Q+(1,0)}$ to $Q, h_{t}(Q)$. For every $t, \operatorname{Ind}\left[h_{t}, C^{*}\right]=\left(\theta_{2}^{t}-\theta_{1}^{t}\right) / 2 \pi \bmod 1$. Since $\operatorname{Ind}\left[h_{t}, C^{*}\right]$ is a continuous function of $t$, and since $\operatorname{Ind}\left[h_{1}, C^{*}\right]=\left(\theta_{2}^{1}-\theta_{1}^{1}\right) / 2 \pi$, we have $\operatorname{Ind}\left[h_{t}, C^{*}\right]=\left(\theta_{2}^{t}-\theta_{1}^{t}\right) / 2 \pi$ for every $t$; in particular it holds for $t=0$. Therefore, $\operatorname{Ind}\left[h, C^{*}\right]=\left(\theta_{2}^{0}-\theta_{1}^{0}\right) / 2 \pi$ where $\theta_{1}^{0}=0$, and $\theta_{2}^{0}$ is strictly between $\pi / 2$ and $3 \pi / 2$. Hence $1 / 4<\operatorname{Ind}\left[h, C^{*}\right]<3 / 4$.

The homeomorphism $\tilde{g}^{-1}$ from $\tilde{g}(\tilde{A})$ onto $\tilde{A}$ satisfies $g^{-1} \circ \pi=\pi \circ \tilde{g}^{-1}$, the homeomorphism $h^{-1}$ is an extension of $\tilde{g}^{-1}$ to the plane, and $h^{-1}$ moves points of $y=0$ to the left and points of $\pi^{-1}(g(\gamma))$ to the right. Applying the procedure of the preceding sections to the homeomorphism $h^{-1}$, we obtain a simple curve $B$ from a point $Z$ in $y \leqslant 0$ to a point $W$ in $\pi^{-1}(g(\gamma))$ which satisfies $-3 / 4<$ $\operatorname{Ind}\left[h^{-1}, B\right]<-1 / 4$. Since $\operatorname{Ind}\left[h^{-1}, B\right]=\operatorname{Ind}\left[h, h^{-1}(B)\right]$, we have $-3 / 4<$ Ind $\left[h, h^{-1}(B)\right]<-1 / 4$. Let $\alpha$ be an arc in $y \leqslant 0$ from $P_{-1}$ to $h^{-1}(Z)$, and let $\beta$ be an arc in $y=F(x)$ from $h^{-1}(W)$ to $Q$. Then each of the $\operatorname{arcs} \alpha+h^{-1}(B)+\beta$ and $C^{*}$ is an arc from $P_{-1}$ to $Q$ which misses any fixed points of $h$. However, $-5 / 4<\operatorname{Ind}\left[h, \alpha+h^{-1}(B)+\beta\right]<1 / 4$, a contradiction of Lemma 8.2.

9. Generalizing the theorem to components of $\operatorname{Fix}(g) .{ }^{3}$ With appropriate modifications of the argument given in the preceding sections, the following result may be obtained:

THEOREM. If $g$ is a twist homeomorphism of the annulus $A$ so that the intersection $F$ of $\operatorname{Fix}(g)$ with the interior of $A$ is a continuum which does not separate the boundary components of $A$, then there is an annular region $R$ which is mapped onto a proper subset of itself by $g$ or $\mathrm{g}^{-1}$, and whose boundary consists either of the unit circle and $a$ simple closed curve in the interior of $A$ which misses Fix $(g)$, or of the unit circle and the union of a subcontinuum of $F$ and a simple open arc in the interior of $A$ which misses $\operatorname{Fix}(g)$.

\footnotetext{
${ }^{3}$ These results suggested by the referee in answer to questions posed by author.
} 
This theorem cannot be strengthened to require the annular region $R$ to have outer boundary a simple closed curve. For example, start with the twist homeomorphism whose flow lines are indicated in Figure 1; expand the one fixed point to a "sin $1 / x$ continuum" of fixed points, modifying the flow lines close to the fixed point as indicated in Figure 7. Any annular region satisfying the conclusion of the theorem must contain the "sin $1 / x$ continuum" as part of its boundary.

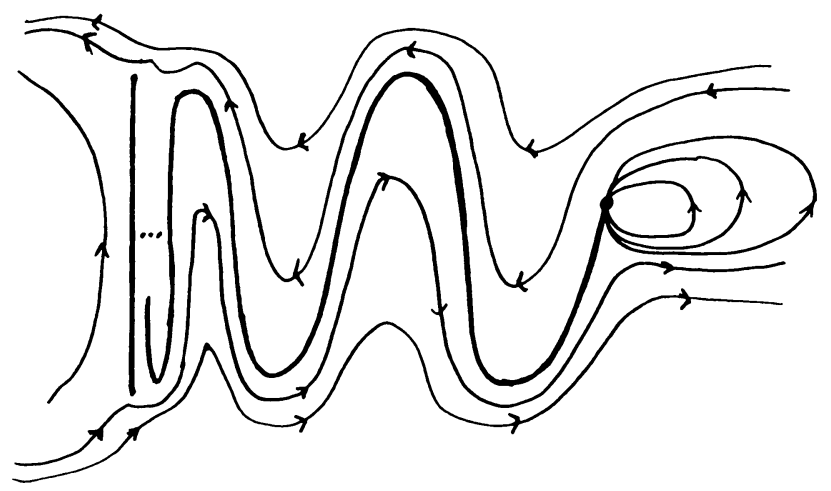

Figure 7

\section{REFERENCES}

1. H. Abelson and C. Stanton, Poincaré's geometric theorem for flows, J. Differential Geom. 11 (1976), 129-131.

2. R. B. Barrar, Proof of the fixed point theorems of Poincare and Birkhoff, Canad. J. Math. 19 (1967), 333-343.

3. G. D. Birkhoff, Proof of Poincare's geometric theorem, Trans. Amer. Math. Soc. 14 (1913), 14-22.

4. An extension of Poincaré's last geometric theorem, Acta Math. 47 (1925), 297-311.

5. , Dynamical systems, Amer. Math. Soc. Colloq. Publ., vol. 9, Amer. Math. Soc., Providence, R. I., 1927.

6. M. Brown and W. D. Neumann, Proof of the Poincare-Birkhoff fixed point theorem, Michigan Math. J. 24 (1977), 21-31.

7. P. Carter, An improvement of the Poincare-Birkhoff Fixed Point Theorem, Dissertation, University of Florida, 1978.

8. C. B. Garcia, A fixed point theorem including the last theorem of Poincare, Math. Programming 8 (1975), 227-239.

9. R. Hermann, Some differential geometric aspects of the Lagrange variational problem, Illinois J. Math. 6 (1962), 634-673.

10. H. Jacobowitz, Periodic solution of $x^{\prime \prime}+f(x, t)=0$ via the Poincaré-Birkhoff theorem, J. Differential Equations 20 (1976), 37-52.

11. , Corrigendum, the existence of the second fixed point: A correction to "Periodic solutions of $x^{\prime \prime}+f(x, t)=0$ via the Poincaré-Birkhoff theorem," J. Differential Equations 25 (1977), 148-149.

12. B. de Kerékjarto, The plane translation theorem of Brouwer and the last geometric theorem of Poincaré, Acta Sci. Math. (Szeged) 4 (1928-1929), 86-102.

13. R. L. Moore, Foundations of point set theory, Amer. Math. Soc. Colloq. Publ., vol. 13, Amer. Math. Soc., Providence, R. I., 1962.

14. W. D. Neumann, Generalizations of the Poincaré-Birkhoff fixed point theorem, Bull. Austral Math. Soc. 17 (1977), 375-389. 
15. M. H. A. Newman, Fixed point and coincidence theorems, J. London Math. Soc. 27 (1952), 135-140.

16. H. Poincaré, Sur un théorème de géométrie, Rend. Circ. Mat. Palermo 33 (1912), 375-407.

17. C. Simon and C. Titus, The fixed point index of symplectic maps, Géométrie Symplectique et Physique Mathématique, Colloq. Internat. C. N. R. S., No. 237, Paris, 1974, pp. 19-28.

18. T. van der Walt, Fixed and almost fixed points, Math. Centre Tracts, Amsterdam, 1963.

Department of Mathematics, University of Maine, Orono, Maine 04469

Current address: 5805 Edson Lane \#304, Rockville, Maryland 20852 